\title{
Reduction of the calcium leaching rate of cement paste by addition of silica nanoparticles
}

\author{
J.J. Gaitero ，I. Campillo , A. Guerrero \\ Labein Tecnalia, Parque Tecnológico de Bizkaia, Edificio 700, $481 ́ 60$ uerw, Spain \\ CIC ncmoGUNE Consolider, Paseo Mikeletegi 56 - 30Í, 20009 San Sebastián, Spain \\ Instituto de ciencias de la construcción "Eduardo Torroja", C/Serrano Galvache 4, 28033 Madrid, Spain
}

Keywords:

B: Calcium silicate hydrate (C-S-H)

Portlandite

Characterization

C: Calcium leaching

D. Nanosilica

\begin{abstract}
A B S TRACT
Calcium leaching is a degradation process that consists in the progressive dissolution of the cement paste as a consequence of the migration of the calcium ions to the aggressive solution. Although it is a well known phenomenon, a way of reducing it has not been found yet. The aim of this work is to prove how the addition of small amounts of silica nanoparticles to the cement paste can induce such reduction. The experimental results obtained have shown that nanosilica increases the strength of the cement paste about a $30 \%$ in cured samples and more than a $100 \%$ in the asymptotically leached ones. At the level of the structure of the C-S-H gel, silica nanoparticles increase the average length of the silicate chains and reduce their polymerization as calcium dissolves. An important decrease of the degradation in terms of porosity and amount of calcium lost has also been observed.
\end{abstract}

\section{Introduction}

The importance of the cementitious materials in the construction industry is nowadays beyond any doubt; however, their variety of applications must not hide their complexity. They are indeed composite materials with truly multi-scale internal structures that keep evolving over centuries. More specifically, the cement paste matrix is basically a porous material composed of calcium hydroxide (portlandite), aluminates and unhydrated cement (clinker) embedded into an amorphous nano-structured hydration product, the so called C-S-H (calcium silicate hydrate) gel . This gel is the most important hydration product of the cement paste, not only because it is the most abundant component (50-70\% by volume), but also because of its exceptionally good mechanical properties Although the C-S-H species are poorly crystalline, an organised structure exists at the nanometric scale, consisting basically of silicate chains of varying lengths held together by layers of calcium. It is this heterogeneous structure, not only of the gel itself but of the whole cement paste, that gives the paste its porous nature. Such pores, of very different sizes and origin, are generally filled with a highly basic solution $(\mathrm{pH}>12.5)$ composed of water and dissolved minerals mainly containing $\mathrm{Ca}(-30 \%$ of the solid mass in cement paste). Therefore, any environment with a $\mathrm{pH}$ lower than this value is aggressive for cement.

Calcium leaching is a degradation mechanism consisting in a progressive dissolution of the cement hydrates as a consequence of the migration of the calcium ions to the pore solution. This phenomenon, which oceurs whenever the paste is put in contact with deionised water, is divided in two stages: dissolution and diffusion. According to Berner's diagrams there is a relationship between the C/S ratio of the hydration producís and the amount of calcium of the pore solution, i.e. the solubility depends on the mineral phase. In this way, Berner distinguishes among three different regimes for the main constituents. Portlandite is only stable when the calcium concentration in the pore solution is higher than $20 \mathrm{~mol} / \mathrm{m}^{3}$. In the case of the C-S-H gel, such equilibrium value can vary between 2 and $20 \mathrm{~mol} / \mathrm{m}^{3}$ depending on its C/S ratio (the higher the C/S ratio of the gel the higher the equilibrium valué). And for calcium concentrations smaller than $2 \mathrm{~mol} / \mathrm{m}^{3}$ only the silicon rich gels, which form at the last stages of calcium leaching, remain stable. Therefore, given a certain concentration, the imbalance and, as a consequence, the dissolution rate will be different depending on the hydrate. This is why the theoretical models identify, at least, two degradation fronts that evolve at different speeds. Furthermore, the response of each of the hydration producís to the attack is also different. While the gel dissolves to precipítate with a lower C/S ratio, leading to a progressive homogenization of the paste, portlandite completely dissociates into $\mathrm{Ca}^{2+}$ and $\mathrm{OH}^{\prime \prime}$, leaving a new pore on its place. 
Although the phenomenon of calcium leaching is nowadays reasonably well understood a way of stopping or, at least, reducing it has not been found yet. Several authors have studied the Caleaching kinetics in cementitious materials processed with silica fume, see for example . Silica fume is composed of silica particles with diameters over $100 \mathrm{~nm}$, i.e. microsilica, whereas silica nanoparticles have typical diameters well below $100 \mathrm{~nm}$. These results in an enhanced surface-to-volume ratio and reactivity leading to different properties as compared to silica fume modified materials . The aim of this article is to shed light on the capabilities of the silica nanoparticles for controlling this decalcification process. As aforementioned, these particles, which could act as both an extremely fine filler and a pozzolan, have been chosen because they have already been successfully used to increase the strength and reduce the curing time of cement preliminary study

Furthermore, it has been reported in a how silica nanoparticles effectively reduce the degradation rate by calcium leaching increasing therefore the durability of the paste. However, to the best of our knowledge, this is the first systematic and thorough study of the effect, on calcium leaching, of the addition of this type of particles to the cement paste. To do so, it has been necessary to prepare a great number of samples which have been characterized by several different techniques that provide information at every scale of matter, from nano, by means of ${ }^{29} \mathrm{Si}$ MAS-NMR and X-ray fluorescence and diffraction, to macro, by compression and bending tests, with the $\mathrm{Hg}$ intrusión porosimetry at an intermedíate level.

\section{Materials and methods}

In order to seize the validity of the silica nanoparticles as a calcium leaching reducing addition, several samples had to be made and analysed. They were prepared at a water-cement ratio $\mathrm{w} / \mathrm{c}=0.4 \mathrm{using}$ an Ordinary Type $152.5 \mathrm{R}$ Portland cement and a $6 \%$ by weight of cement of four different types of commercial silica nanoparticles, see Table 1. The mixing process varied depending on the presentation of the nanoparticles as a colloidal dispersion (CS) or a dry powder (ADS). While in the first case the dispersión was mixed with the water and stirred for $5 \mathrm{~min}$ before adding the solution to the cement, in the other one the powder was mixed with the cement and agitated for a minute before pouring the water on it. One set without silica nanoparticles was also prepared for comparison. The samples were cast into $1 \times 1 \times 6 \mathrm{~cm}$ prisms using steel moulds, where they stayed for $24 \mathrm{~h}$ in a chamber at $20{ }^{\circ} \mathrm{C}$ and $100 \%$ humidity. After that time, they were unmoulded and introduced in a saturated lime solution to prevent any premature loss of calcium during the remaining 27 days of curing. At the end of this period, they were moved into a new bath containing the aggressive solution. The slow evolution of the leaching process made it necessary to use an accelerated method consisting of immersing the samples into a $6 \mathrm{M}$ ammonium nitrate solution that increases the leaching rate by a factor of 300 compared to the immersion in deionised water . The best quality of this method is that, in addition to its important acceleration rate, it does not alter the nature of the degradation process, which consists of a dissolution

Table 1

Main physicochemical properties of the commercial additions used as stated by the manufacturer

\begin{tabular}{llllll}
\hline$\tilde{N}$ Name & Particle size $(\mathrm{nm})$ & $\mathrm{pH}$ & Stabilizing agent & $\mathrm{Si0}_{2}$ content $(w t \%)$ & Presentation \\
\hline CS1 & 30 & 10 & $\mathrm{Na}_{2} \mathrm{O}$ & 45 & Colloid \\
CS2 & 20 & 10 & $\mathrm{Na}_{2} \mathrm{O}$ & 20 & Colloid \\
CS3 & 120 & 9.5 & $\mathrm{NH}_{3}$ & 40 & Colloid \\
ADS & $1400^{\mathrm{a}}$ & - & - & 95 & Powder \\
\hline
\end{tabular}

All the colloids were dispersed in water being the amount of the stabilizing agents $<0.1$ wt. $\%$.

a The given valué corresponds to the agglomerated particle size; the individual particle size is $15 \mathrm{~nm}$. followed by a diffusion, as it was mentioned before. The only difference is that the higher solubility of calcium in this solution increases the imbalance and therefore the dissolution rate. In order to prevent the samples from carbonation, the aggressive bath was maintained all the time in contact with a nitrogen atmosphere and to ensure its aggressiveness the solution was renewed whenever its $\mathrm{pH}$ reached a valué of 9.2. Gentle shaking was also applied to homogenize the solution.

One of the key parameters in calcium leaching, like in any other degradation process, is the time of exposition to the aggressive solution. Therefore, a tight schedule was created to follow the evolution of the samples as a function of time. The tests were systematically performed 7 and 28 days after casting of the samples ( $\mathrm{t}_{-}$! and $\mathrm{t}_{0}$ respectively), still within the curing process, and 9, 21, 42 and 63 days after the immersion in the aggressive solution $\left(\mathrm{ti}, \mathrm{t}_{2}, \mathrm{t}_{3}\right.$ and $t_{4}$ respectively). Using this sequence, it is possible to study separately the rapid dissolution of portlandite and the slow decalcification of the C-S-H gel, which do not reach the level of asymptotic degradation before $t_{3}$. Exceptionally, some additional experiments were carried out at other ages to complete the information. Due to the complexity of the studied phenomenon several different techniques had to be used to thoroughly characterize the material at every scale of matter.

Two of the most important tests from an engineering point of view are the compression and bending tests. In spite of being the only two macroscopic methods used in this work, they provide the parameters that assess the validity of the material for its application in construction. The equipments used for this study were tailor made to meet the requirements of the samples in terms of dimensions and strength. They were just reproductions of the standardised ones but at a smaller scale. Therefore, all the testing procedure was analogous to the one presented in the norm

Another very important parameter in terms of both strength and prevention of degradation is the porosity . This was measured using a Micromeritics Autopore 111 mercury intrusión porosimetre which is capable of measuring the volume of pores with diameters in the range $7 \mathrm{~nm}-350 \mathrm{um}$, i.e. the capillary (10 nm-5 um) and macroscopic ( $>5 \mathrm{um}$ ) porosity of the undegraded cement paste. The latter are generally spherical and attributed to the presence of entrapped air because of improper vibration, while the former, by contrast, refer to the capillary voids that appear between cement grains during hydration as a consequence of the lower average bulk density of the hydration producís compared to the cement grains Once the degradation commences, two new types of pores begin to appear: the ones corresponding to the total dissolution of portlandite (from 1-300 um) and the ones arising from the decalcification of the rest of hydrates, which dissolve to precipitate with a lower C/S ratio. In spite of being one of the most popular techniques for the pore size analysis in ceramic materials, mercury porosimetry has two important shortcomings: it requires drying of the specimen and assumes that pores are cylindrical. All in all, Diamond showed that, although it tends to shift the pore size distribution towards the smaller pore sizes, the measurement of the total porosity is reasonably accurate.

Portlandite consumption as a consequence of both calcium leaching and reaction with nanosilica, as well as the evolution of the rest of the crystalline phases present, was measured using a Philips XPert Pro X-ray diffractometer. This apparatus uses the method of the powder, or of Debye Scherrer, in which the sample is introduced in the form of a powder. Sample preparation consisted simply in grounding a representative piece of the paste and then compacting it manually; therefore, the results can be considered as an average. But this is not the only X-ray based technique working with this kind of samples that has been used in this work. X-ray fluorescence has been employed to quantify the relative amounts of the oxides of the ten principal elements present and sulphur.

The only experimental technique specifically chosen to study the internal structure of the $\mathrm{C}-\mathrm{S}-\mathrm{H}$ gel is ${ }^{29} \mathrm{Si}$ MAS-NMR . This 


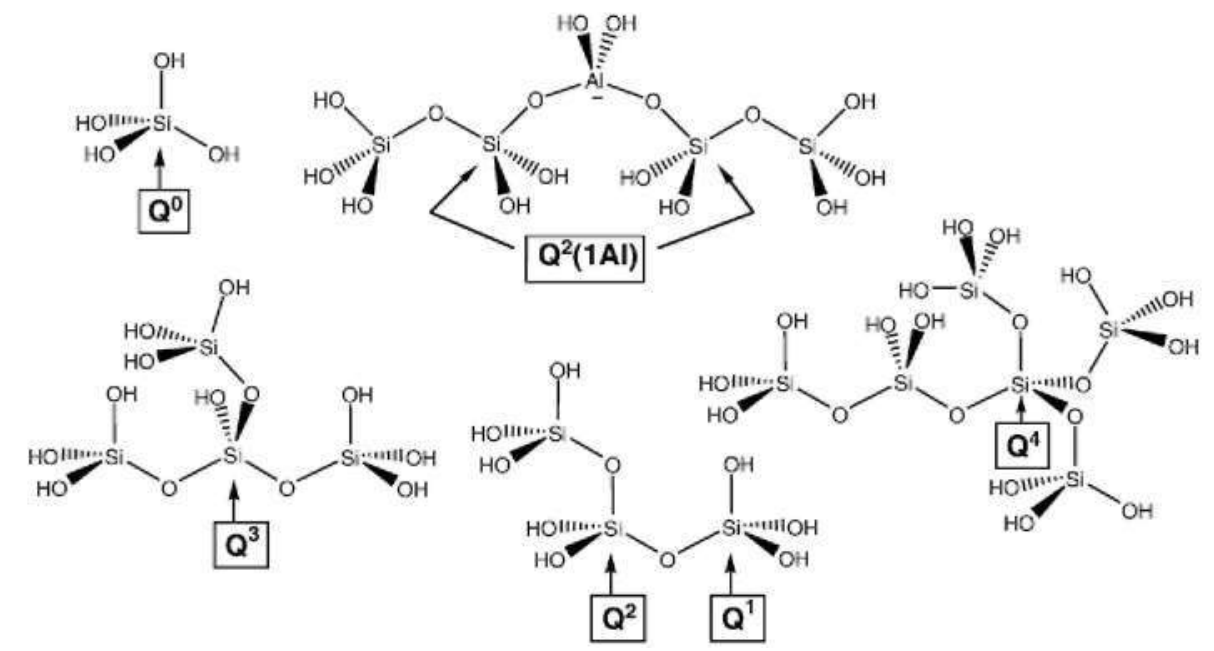

Fig. 1. Diagrams of the different types of silicate chains present in the C-S-H gel and their nomenclature with résped to NMR analysis.

technique, whose test specimens were prepared following the same procedure used for the X-ray diffraction tests, gives information about the connectivity of each of the silicon atoms present in hydrated cement, which can be connected to $0,1,2,3$ or $4 \mathrm{Si}$ atoms by an $\mathrm{O}$ atom. Each of these connections gives place to a different peak $\left(Q^{\circ}, Q^{1}, Q^{2}, Q^{3}\right.$ and $\mathrm{Q}^{4}$ respectively) in the NMR spectrum, see Fig. 1, plus the peak $\mathrm{Q}^{2}$ $(1 \mathrm{Al})$ corresponding to the connection with a silicon and an aluminium atom. From the áreas of the peaks it is possible to define the degree of hydration $(\mathrm{H})$, the average segment length $(<\mathrm{SI}\rangle)$ and the degree of polymerization $(\mathrm{P})$ as

$$
H=\frac{Q^{1}+Q^{2}+Q^{3}+Q^{4}}{Q^{0}+Q^{1}+Q^{2}+Q^{3}+Q^{4}}
$$$$
\langle\mathrm{SI} .\rangle-\frac{2\left(Q^{1}+Q^{2}+1.5 Q^{2}(1 \mathrm{Al})+3 Q^{3}+4 Q^{4}\right)}{Q^{1}+3 Q^{3}+4 Q^{4}}
$$

$$
\mathrm{P}=\frac{Q_{-^{\prime}+Q^{4}}}{Q\}+Q ?+I .5 Q ?(I M)+Q\}+Q ?^{\prime}}
$$

where $\mathrm{Q}^{1}$ represents the área of the peak $i$.

While the degree of hydration has already been defined elsewhere

, the average segment length is a generalization of the average chain length, valid only for linear chains (formed by $\mathrm{Q}^{1}$ and $\mathrm{Q}^{2}$ type connections), to the case containing also planar and three-dimen- sional structures (including also atoms with $\mathrm{Q}^{3}$ and $\mathrm{Q}^{4}$ type connectivities). $\langle\mathrm{SI}\rangle$ represents the number of length units, i.e. silicate and/or alumínate tetrahedra, between a $Q^{1}$ and a $Q^{3}$, a $Q^{1}$ and a $\mathrm{Q}^{4}$, a $\mathrm{Q}^{3}$ and a $\mathrm{Q}^{4}$, and two $\mathrm{Q}^{1}, \mathrm{Q}^{3}$ or $\mathrm{Q}^{4}$ type atoms, extremes included. On the other hand, the polymerization is a measure of the mean number of crossings $\left(\mathrm{Q}^{3}\right.$ and $\left.\mathrm{Q}^{4}\right)$ by tetrahedron. The equipment used for this study was a Bruker Avance DSX 300 working with single pulses of 4.6 us, with a delay time of $3 \mathrm{~s}$ and the sample rotating at $7200 \mathrm{~Hz}$. The reference was set at $6.65 \mathrm{ppm}$ using hexamethyldisiloxane and the analysis of the data was made with the program Brukerl D WTN-NMR provided with the equipment.

The usual way of evaluating the mechanical performance of cement-based materials is by means of the compression and bending tests. The first thing that becomes apparent from the results obtained in such tests, see Fig. 2, is the different behaviour of the REF specimens with respect to the ones containing nanosilica. After only 7 days of curing $(t-i)$, the increase in compressive strength induced by the nanoparticles is already appreciable, rising this value up to a $20-30 \%$ at $t_{0}$ (after 28 days of curing). However, this difference becomes even bigger once the degradation begins, because, while at ti the REF and ADS samples undergo a reduction of strength of $75 \%$ and $65 \%$ respectively, in the case of the ones containing the colloidal dispersions this reduction is only about $10 \%$. As the degradation goes on, the gap between the two types of nanoparticles becomes less
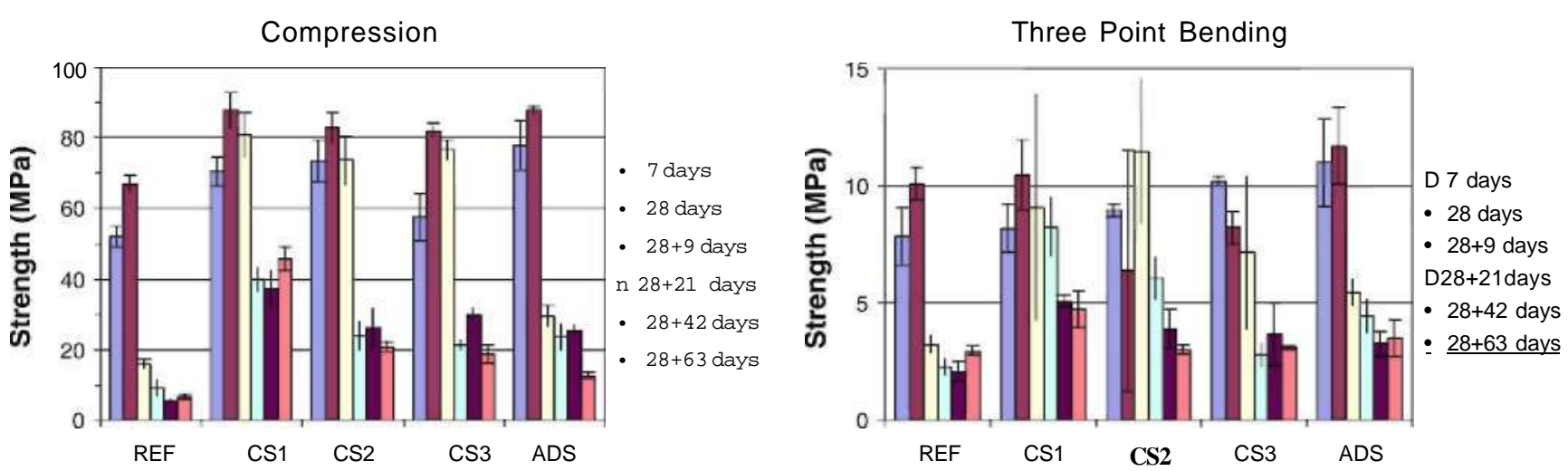

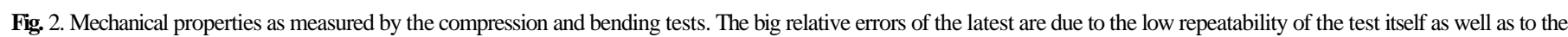
smaller number of specimens studied [20], three. 

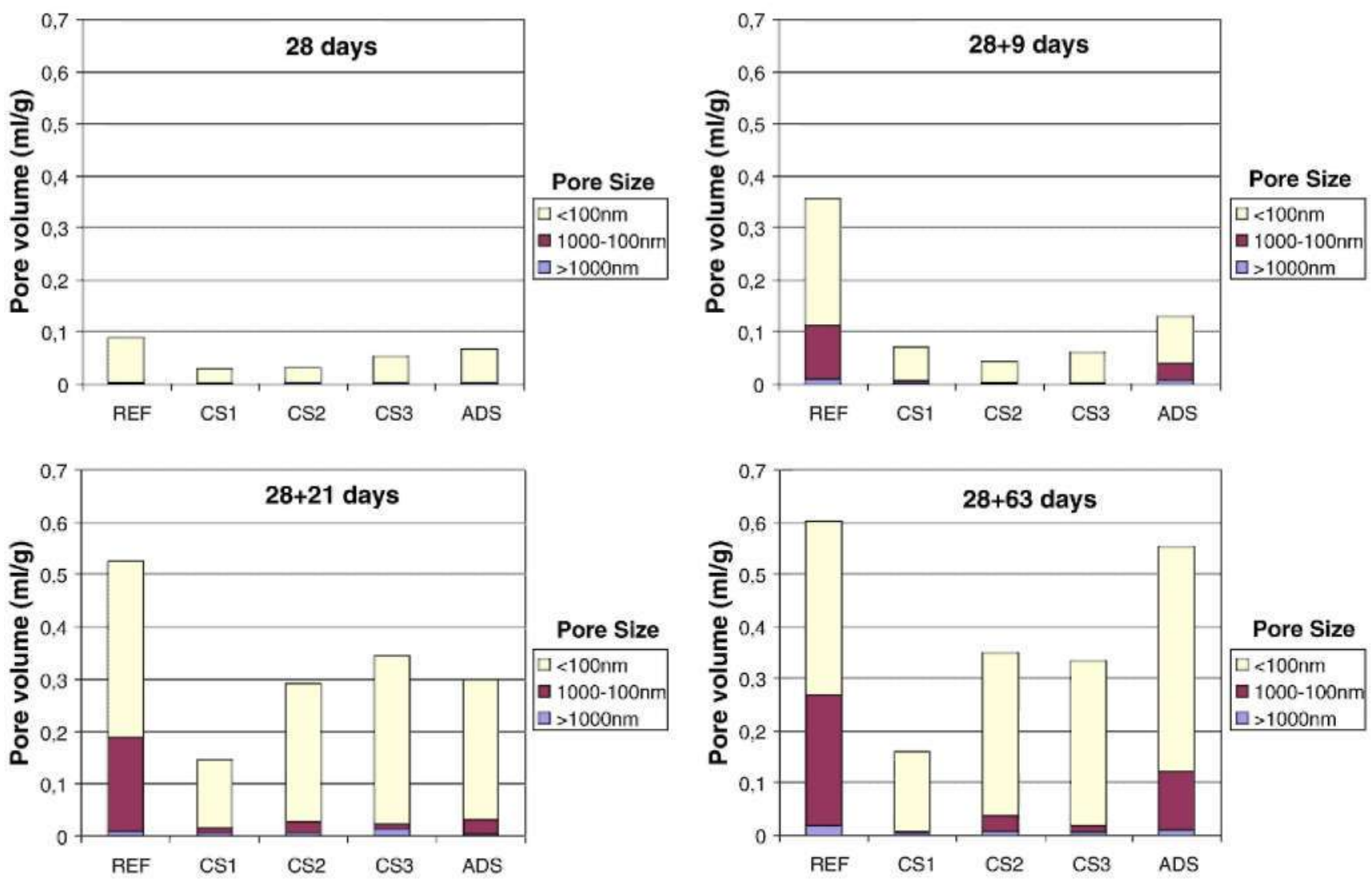

Fig. 3. Total pore volume and pore diameter distribution measured by mercury intrusión porosimetry assuming a cylindrical geometric representation.

evident but not the one with respect to the reference (REF) that at the end of the study $\left(\mathrm{t}_{4}\right)$ has a compressive strength half that of the ADS sample, one third that of the CS2 and CS3 and about 7 times smaller than the one of the CS1. On the other hand, the data obtained from the bending tests are far more irregular due to the high sensitivity of this experiment to the imperfections of the test specimens and the smaller number of samples used (only three) . As a consequence, it is very difficult to extract any conclusión prior to $t_{v}$ After this point, the results follow a tendency similar to the one of the compression data but with much smaller differences among samples at the end of the study.

Due to the nature of calcium leaching, involving simultaneous phenomena of dissolution and diffusion, it is evident that the results presented above must be greatly influenced by the variations of porosity, see Fig. 3. The first consequence of the introduction of the silica nanoparticles is the reduction of the porosity at $t_{0}$, although the most important effects become apparent once the degradation begins. At $t i$, the pore volume of REF has already increased by a factor of 4 , while such factor reduces down to 2 in the ADS and even further in the others. This tendency is maintained along time but at a much slower rate, until REF almost stabilizes at $t_{4}$ in very good correlation with the compression results. In addition, analysing the pore size distribution during the degradation process, it is possible to observe how this is also different in REF than in the other samples. While in the former half of the total pore volume at $t i$ is on the range 1-0.1 um, in the others this accounts for less than the $25 \%$ and even less at later stages.

According to the two front model developed by Heukamp and Ulm, portlandite should have completely disappeared after about seven days of accelerated calcium leaching . The results of the X-ray diffraction study at $t i$, see Fig. 4, confirm this prediction but only in two samples, REF and ADS, which is one of the reasons for their high porosity at this age. One of the possible explanations for this can also be found on the porosity, see Fig. 3, which was smaller on the other three specimens making it more difficult for the aggressive solution to get into the samples. However, far more information can still be extracted from the X-ray spectra. The semi-quantitative measurement of the amount of portlandite at $t-i$ and $t_{0}$ is a good indicator of the activity of the silica nanoparticles, as these particles react with the calcium hydroxide of the paste to produce C-S-H gel. According to such results, see Fig. 4, the colloidal silica nanoparticles CS1 and CS2 are far more reactive than CS3 and the agglomerated dry silica, which can be attributed to the difference in particle sizes. Nevertheless, at $t_{B}$ all four of them have similar amounts of calcium hydroxide, which indicates that both types of nanoparticles have reacted in a comparable proportion. Furthermore, taking into account the small amount of silica nanoparticles used, the high degree of hydration of the paste at $t_{0}$ and the fast dissolution of portlandite in ammonium

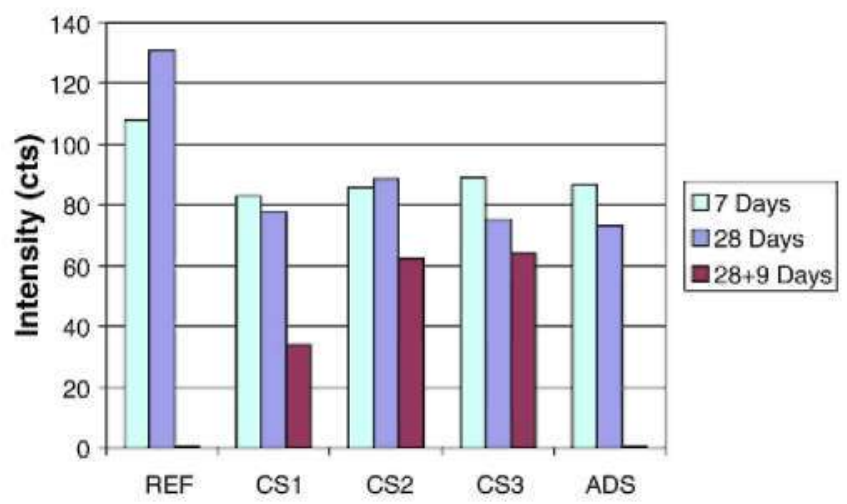

Fig. 4. Intensity of the portlandite peak placed at $28=18.05^{\circ}$ as obtained by the method of the powder. The valúes presented here are semi-quantitative; however, they are valid for comparison as they were all measured and analysed under the same conditions. 
(a)

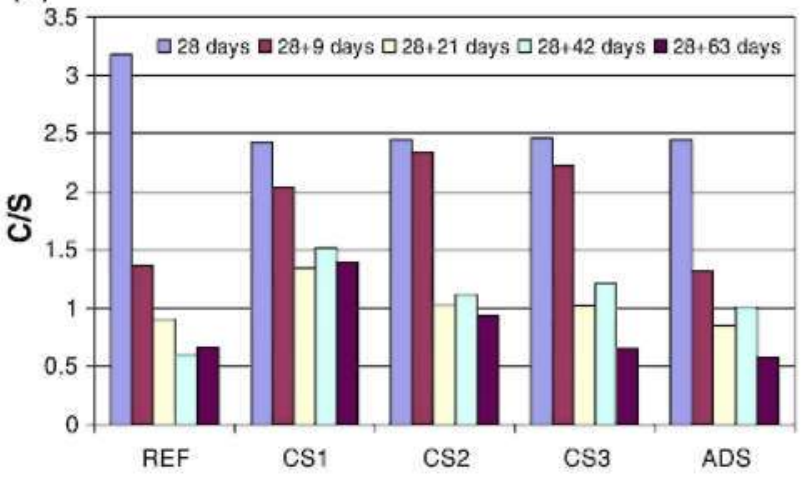

(b)

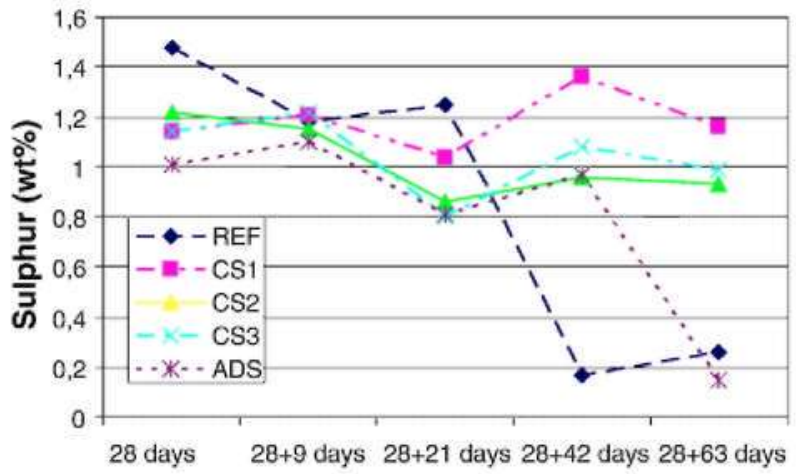

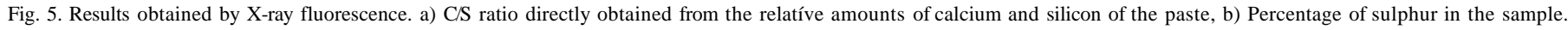

nitrate, it is possible to assume that any reduction of calcium hydroxide during the degradation process is just a consequence of the degradation and not of the reaction with the silica nanoparticles. This will be further reinforced by the NMR results, see below, which show no evidence of the presence of unreacted silica nanoparticles after 28 days of curing.

Another way of following the dissolution of calcium hydroxide, altogether with the degradation of the rest of hydrates, is in terms of the C/S ratio, which can be obtained from the X-ray fluorescence measurements. Although this technique gives average information, separation of both phenomena is relatively easy, at least in the samples REFand ADS, due to the great differences in degradation rates between them. The approximation followed in this work is that all the calcium lost at $\mathrm{ti}\left(\mathrm{t}_{2}\right.$ for samples CS1, CS2 and CS3) is a consequence of the dissolution of portlandite, while after that time only the C-S-H gel is left. This approximation is supported by the results of X-ray diffraction which show no evidence of the presence of portlandite after $t_{t}$ in REF and ADS, see Fig. 4 ( $t_{2}$ for samples CS1, CS2 and CS3). The results presented in Fig. 5a show how at $t_{0}$ the $\mathrm{C} / \mathrm{S}$ ratio is much greater in REF than in the other samples. Although this data was already known as a consequence of the addition introduced in such samples, it serves as a check of the accuracy of the measurements, always with an error smaller than $10 \%$. As soon as the degradation process begins, the sample REF commences its fast calcium loss until it stabilizes at $t_{3}$. At this point the sample is already asymptotically leached, being its C/S valué in good agreement with the ones reported in the literature . On the contrary, CS2, CS3 and ADS evolve much

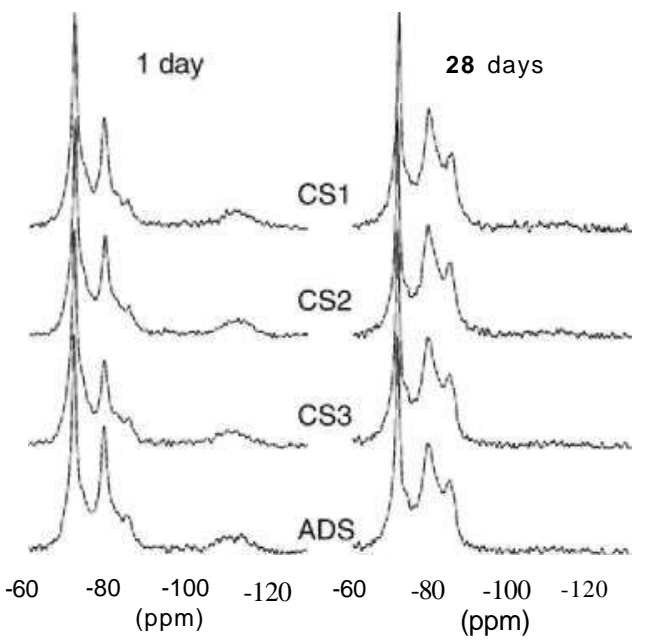

Fig. $6 .{ }^{29} \mathrm{Si}$ MAS-NMR spectra of the samples containing silica nanoparticles after 1 (left) and 28 (right) days of curing. The wide peak at $-110 \mathrm{ppm}$ of the 1 day spectra corresponds to the presence of unreacted nanosilica. more slowly and do not reach a similar level until $\mathrm{t}_{4}$. However, due to the extra amount of silicon introduced with the additions, this apparent equality is not completely real because it indicates a smaller calcium loss. Anyway, once the degradation is so severe that calcium dissolution cannot restrain equilibrium in the pore solution other elements like sulphur have to take its place, see Fig. 5b. Finally, it is worth mentioning the special behaviour of CS1 which gives the bests results in every measurement after ti, showing no calcium loss after this point. Note also the good correlation of these data with the results of the mechanical tests.

${ }^{29} \mathrm{Si}$ MAS-NMR is becoming more and more common in cementitious materials research. Here, it was primarily used to evalúate whether the introduction of silica nanoparticles into the cement paste had any effect in the internal structure of the C-S-H gel, see Fig. 8; however, it also provides information about the degree of hydration of the paste (Fig. 7), as well as, about the presence or not of unreacted silica nanoparticles (Fig. 6). As can be observed in Fig. 6, although such unreacted nanosilica is clearly present $\left(Q^{4}\right.$ peak - $\left.110 \mathrm{ppm}\right)$ after one day of curing, there is no evidence of its presence at the end of the curing process $t_{0}$, what reinforces the assumption that any portlandite consumption posterior to $t_{0}$ is just a consequence of the degradation. Fig. 7 shows how, at $t_{0}$, all the samples are similarly hydrated; however, this changes radically as soon as degradation begins. While at ti the samples REF and ADS are already completely hydrated, the other three have barely evolved and it takes to the samples CS2 and CS3 21 days of accelerated calcium leaching $\left(\mathrm{t}_{2}\right)$ to reach a similar level and even longer to CS1. Actually, an important amount of unhydrated cement has been detected in the sample CS1 even after 63 days of exposure to the aggressive solution $\left(t_{4}\right)$. This is definitely one of the reasons of its outstanding mechanical properties because unreacted

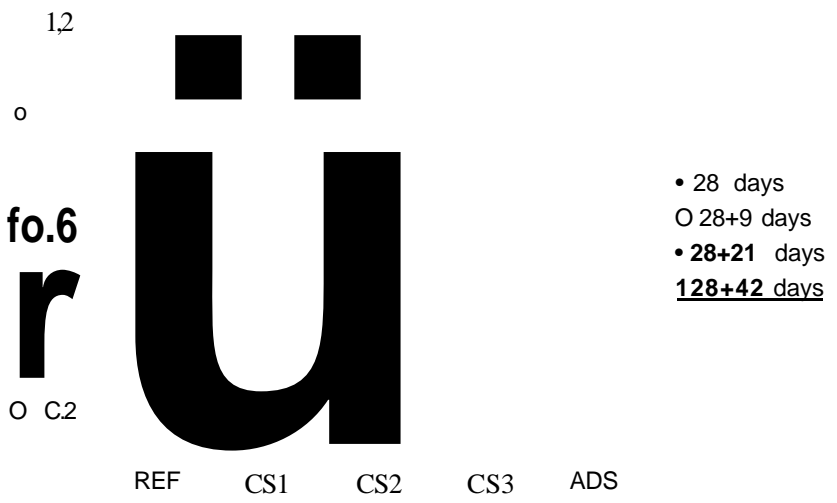

Fig. 7. Degree of hydration obtained from the relative áreas of the ${ }^{29}$ Si MAS-NMR spectra according to the deflnition presented above. 
(a)

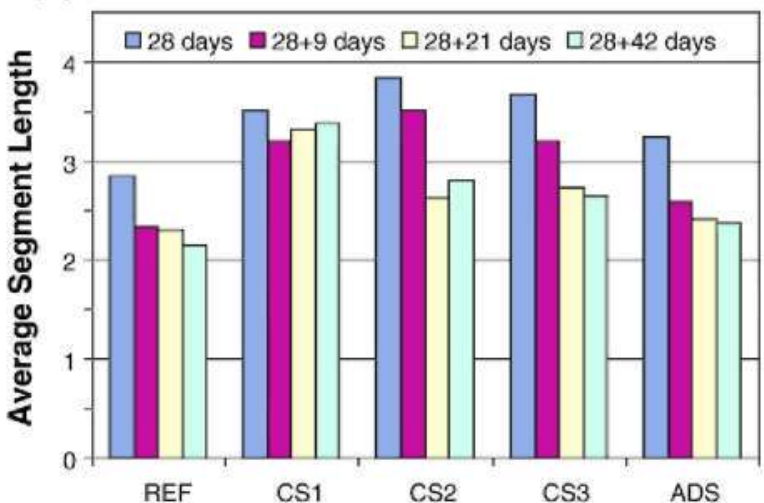

(b)

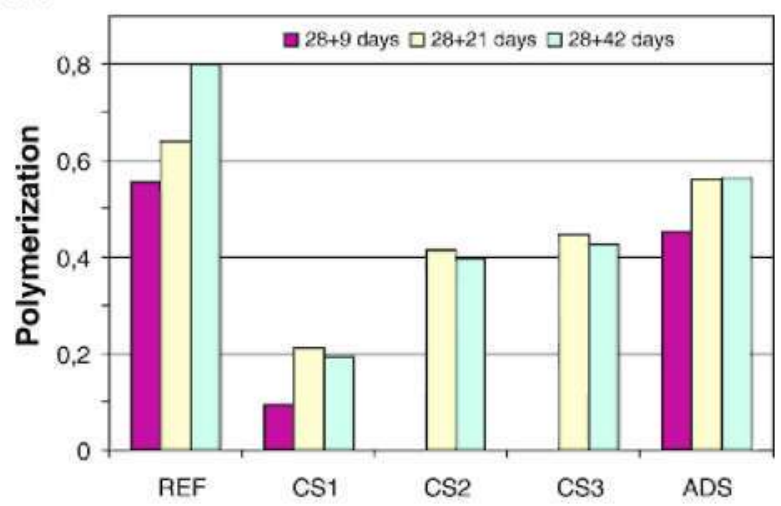

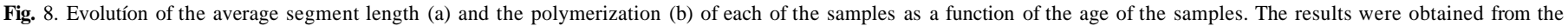
relative áreas of the ${ }^{29} \mathrm{Si}$ MAS-NMR spectra applying the equations deflned above.

cement hydrates in contact with the aggressive solution replacing part of the dissolved material. Therefore, the presence of the clinker indicates that there is a región which has barely been degraded and surrounding it an área that, in spite of having been degraded, it has been reinforced by new hydration producís. The reason of the preservation of such unreacted cement lies partly on the porosity, see Fig. 3, as the three samples with colloidal silica have a lower porosity than the others at $\mathrm{t}_{0}$, making it more difficult for the aggressive solution to get into them. However, this does not account for the different evolution of the sample CS1 compared to the CS2 and CS3, what should be further studied. Focusing now on the structure of the C-S-H gel, it can be observed in Fig. 8a how the addition of silica nanoparticles to the cement paste favours the growth of the silicate chains, increasing their average length in spite of the smaller degree of hydration. Furthermore, although as soon as the degradation begins the average segment length $(<\mathrm{SI}\rangle)$ falls in every case, its value is always greater in the samples with nanosilica than in REF. The behaviour of the degree of polymerization is exactly the opposite, see Fig. 8b, corresponding the lowest valúes to CS1 and the highest to REF. This good correlation is very remarkable because it indicates that longer chains correspond to more stable C-S-H.

\section{Conclusions}

A coherent set of data from different characterization techniques has been collected on the study of calcium leaching phenomenon in cement paste matrices with silica nanoparticles. The use of an accelerated degradation method, the prolonged time of exposure to the aggressive solution and the relatively small samples have made possible to study the whole decalcification process until the asymptotic leaching state. The obtained results have shown that the introduction of the silica nanoparticles modifies the cement paste in three different ways:

- reducing the porosity.

- transforming portlandite into C-S-H gel by mean of a pozzolanic reaction.

- modifying the internal structure of the C-S-H gel increasing the average chain length of the silicate chains.

It seems that it is the sum of all of these effects that is responsible for the good performance of the silica nanoparticles as a calcium leaching controlling addition. The reduction of porosity makes it more difficult for the aggressive solution to get into the samples slowing down the degradation rate. The pozzolanic reaction transforms calcium hydroxide, which is the phase most susceptible of calcium leaching, into C-S-H gel, improving the mechanical performances and making the paste more resistant to the chemical attack. And finally, the modification of the internal structure of the gel towards longer silicate chains increases calcium stabilization.

The study of the degradation itself has shown how, apart of the increase of porosity and the loss of mechanical strength of the paste, the migration of calcium ions has an effect on the internal structure of the C-S-H gel and in the unreacted clinker which changes with the introduction of the nanoparticles. More specifically, the reduction of the C/S ratio of the gel induces a rearrangement of the silicate chains that polymerize giving place to more connected structures. This phenomenon that appears in the reference samples (REF) as soon as the degradation begins is greatly reduced in the modified ones, what indicates that less calcium has been lost in the latest. The same happens with the hydration of the unreacted clinker. While in the reference sample (REF) this disappears at the initial stages of the degradation, it takes much longer in the modified ones what, on the one hand, is a sign of the slower degradation and, on the other hand, helps to slow it further as it will hydrate as a consequence of the degradation itself taking the place of the lost material.

It can be concluded that the addition of nanosilica to cement-based materials can control C-S-H degradation induced by Ca-leaching at the same time that it increases the overall strength at every age. Nevertheless, the magnitude of these beneficial effects depends on the presentation of the nanoparticles, being more important in the colloidal dispersions than in the dry ones.

\section{Acknowledgements}

This work has been supported by the Department of Industry, Trade and Tourism of the Basque Government under the ETORTEK IE05-151 and SAIOTEK S-PE06LA05 projects, and by the Spanish Ministery of Science and Education under the MONACEM project (Ref MAT2005-0390) and the Consolider-Ingenio 2010 Program "CIC nanoGUNE Consolider" CSD2006-53.

\section{References}

H.F.W. Taylor, Cement Chemistry, Thomas Telford, London, 1997.

G. Constantinides, F.J. Ulm, The nanogranular nature of C-S-H, J. Mech. Phys. Solids 55 (2007) 64-90.

I.G. Richardson, G.W. Graves, Models for the composition and structure of calcium silicate hydrate $(\mathrm{C}-\mathrm{S}-\mathrm{H})$ gel in hardened tricalcium silicate pastes, Cem. Concr. Res. 22 (6) (1992) 1001-1010

H.F.W. Taylor, Proposed structure for calcium silicate hydrate gel, J. Am. Ceram. Soc. 69 (6) (1986) 464-467

U.R Berner, Modeling the incongruent dissolution of hydrated cement materials, Radiochim. Acta 44/45 (1988) 387-393.

M. Mainguy, O. Coussy, Propagation fronts during calcium leaching and chloride penetration, J. Eng. Mech. ASCE 126 (3) (2000) 250-257. 
M. Mainguy, C. Tognazzi, J.M. Torrenti, F. Adenot, Modelling of leaching in puré cement paste and mortar, Cem. Concr. Res. 30 (1) (2000) 83-90.

FJ. Ulm, J.M. Torrenti, F. Adenot, Chemoporoplasticity of calcium leaching in concrete, J. Eng. Meen. ASCE 125 (10) (1999) 1200-1211.

F. Adenot, M. Buil, Modeling of the corrosión of cement paste by deionized water, Cem. Concr. Res. 22 (2-3) (1992) 489-496.

S. Kamali, B. Gérard, M. Moranvüle, Modelling the leaching kinetics of cementbased materials-influence of materials and environment, Cem. Concr. Compos. 25 (4-5) (2003) 451-458.

J.J. Chen, J.J. Thomas, H.M. Jennings, Decalciflcation shrinkage of cement paste, Cem. Concr. Res. 36 (5) (2006) 801-809.

C. Porteneuve, H. Zanni, C. Vernet, K. Kjellsen, J.P. Korb, D. Petit, Nuclear magnetic resonance characterization of high- and ultrahigh-performance concrete application to the study of water leaching, Cem. Concr. Res. 31 (12) (2001) 1887-1893.

V. Matte, M. Moranvüle, Durability of reactive powder composites: influence of silica fume on the leaching properties of very low water/binder pastes, Cem. Concr. Compos. 21 (1) (1999) 1-9.

J. Bjornstrom, A Martinelly, A. Matic, L Borjesson, I. Panas, Accelerating effeets of colloidal nano-silica for beneflcial calcium-silicate-hydrate formation in cement, Chem. Phys. Lett. 392 (1-3) (2004) 242-248.

1. Campillo, J.S. Dolado, A. Porro, High performance nanostructured materials for construction, Proceedings of the 1st International Symposium on Nanotechnology in Construction, Paisley (Scotland), 2003, pp. 215-225.

A Porro, J.S. Dolado, I. Campillo, E. Erkizia, Y.R de Miguel, Y. Saez de Ibarra, A Ayuela, Effeets of nanosilica additions on cement pastes, Applications of Nanotechnology in Concrete Design, Thomas Telford, London, 2005, pp. 87-95.
J.J. Gaitero, Y.S. de Ibarra, E. Erkizia, I. Campillo, Silica nanoparticle addition to control the calcium-leaching in cement-based materials, Phys. Status Solidi 203 (6) (2006) 1313-1318.

H. Saito, S. Nakane, S. Ikari, A Fujiwara, Preliminary experimental study on the deterioration of cernentitious materials by an acceleration method, Nucí. Eng. Des. 138 (2) (1992) 151-155

F. Heukamp, FJ. Ulm, J. Germaine, Mechanical properties of calcium leached cement pastes: triaxial stress states and the influence of the pore pressure, Cem. Concr. Res. 31 (5) (2001) 767-774.

UNE-En 196-1 2005.

K. Haga, S. Sutuou, M. Hironaga, S. Tanaka, S. Nagasaki, Effeets of porosity on leaching of $\mathrm{Ca}$ from hardened ordinary Portland cement paste, Cem. Concr. Res. 35(9)(2005)1764-1775

M. Pigeon, The frost durability of concrete, Cement and Concrete Science and Technology Vol. 1, Part II, Abi Books (P) LTD, New Delhi, India, 1992, pp. 417-448. S. Diamond, Aspects of concrete Porosity Revisited, Cem. Concr. Res. 29 (8) (1999) 1181-1188.

X. Cong, RJ. Kirkpatrick, ${ }^{29} \mathrm{Si}$ MAS NMR study of the structure of calcium silicate hydrate, Adv. Cem. Based Maten 3 (3/4) (1996) 144-156.

I.G. Richardson, The nature of C-S-H in hardened cements, Cem. Concr. Res. 29 (8) (1999)1131-1147.

C. Carde, R Francois, J-M. Torrenti, Leaching of both calcium hydroxide and C-S-H from cement paste: modeling the mechanical behavior, Cem. Concr. Res. 26 (8) (1996) 1257-1258. 